\title{
Nonlinearity of interfaces and force transmission of bolted flange joints under impact loading
}

\author{
Yacong Guo ${ }^{\mathrm{a}, \mathrm{b}}$, Yanpeng Wei ${ }^{\mathrm{a}, \mathrm{b}, *}$, Zhe Yang $^{\mathrm{a}, \mathrm{b}}$, Chenguang Huang ${ }^{\mathrm{a}, \mathrm{b}}$, Xianqian $\mathrm{Wu}^{\mathrm{a}, \mathrm{b}}$, \\ Qiuyun Yin ${ }^{\mathrm{a}, \mathrm{b}}$
}

${ }^{a}$ Key Laboratory for Mechanics in Fluid Solid Coupling Systems, Institute of Mechanics, Chinese Academy of Sciences, Beijing 100190, China

${ }^{\mathrm{b}}$ School of Engineering Science, University of Chinese Academy of Sciences, Beijing, 100049, China

\section{A R T I C L E I N F O}

\section{Article History:}

Received 6 January 2017

Revised 1 April 2017

Accepted 29 June 2017

Available online 30 June 2017

\section{Keywords:}

Bolted flange joint

Vibration

Shock response

Impact load

Interfaces

Force transmission

\begin{abstract}
A B S T R A C T
Vibration of flange joints and force transmission characteristics of connection interfaces are important due to concerns over the structural strength and signal identification in engineering structures such as missiles and rockets. However, nonlinear factors such as clearance, damping and discontinuous interfaces make it difficult to predict the responses under specific excitations such as shock impacts. This paper proposed a typical configuration of a bolted flange joint and investigated the response of vibration and dissipation of the joint under a series of impact loadings. The amplitude of excitation was $120 \mathrm{MPa}$, and the duration of the loading ranged from $20 \mu \mathrm{s}$ to $200 \mu \mathrm{s}$. The excitations of high amplitude and frequency lead to a vibrational response coupled to a shock response of the joints. The connecting interfaces contributed to the distortion of the shockwave and changed its frequency, which can be characterized by the factor $\alpha$. Those interfaces were also sources of energy dissipation, and their effect decreased as the frequency of excitation decreased. This methodology is helpful for predicting the local behavior of joints in a complex engineering structure under extreme conditions.
\end{abstract}

(C) 2017 Elsevier Ltd. All rights reserved.

\section{Introduction}

A bolted flange joint is a common connection used in engineering structures such as aircraft engines, pipelines and projectiles due to their ease of assembly, operation and portability. The configuration usually consists of several bolts connecting a base and a flange. Due to the existence of the bolt-screw joint, structural damping and discontinuous interfaces, the force transmission is nonlinear and the vibration of the flange is much more complicated and difficult to predict. Therefore, a method for investigating the interfaces and dynamic response is required.

Much research has been conducted on the nonlinearity of bolted joints subjected to static forces. Cao and Bell [1] proposed a formula to calculate the nonlinear bolt force caused by clearances when the flange joint was under tension. Characteristic parameters of a flange and bolt including material and geometry properties were evaluated to fully understand the regulation. Bickford [2] introduced a prying force to explain non-uniform contact of interfaces under tension. It was determined that the nonlinearity mainly comes from different stiffness under tension and compression, as well as the prying effect

\footnotetext{
Corresponding author.

E-mail address: weiyanpeng@imech.ac.cn (Y. Wei).
}

[3]. Linear systems have been widely used for simple modeling of the joints in previous studies due to their high efficiency. A springmass system was used to predict the force and motion transmissibility ratio between bolted flange joints, aiming to optimize the design of preloaded bolted joints under harmonic excitation [4]. The stiffness of the flange and bolt were investigated separately, though further validation is required when the $2 \mathrm{D}$ model is used for a $3 \mathrm{D}$ structure. Mackerle [5] suggested that research in bolted connections should be simulated with 3D modeling rather than 2D modeling, which gave stiffer and stronger results.

A linear system used for modeling the real bolted flange joint is imprecise since many factors, including material and geometrical nonlinearities, contact, friction and bolt-flange interaction, contributed to nonlinearity [5]. Furthermore, it is difficult to find a uniform standard for analyzing bolted flange joints under dynamic impact [6], including complex geometry and material properties, nonlinear dynamic algorithm and low computational cost. Several different nonlinear models are applied to simulate the response of bolted joints. Oldfield et al. [7] proposed a finite element model using Jenkin's elements and the Bouc-Wen model to investigate energy dissipation. They conducted dynamic frictional contact under harmonic loading and compared the difference between the two models. Ouyang et al. [8] gave further validation for the Jenkins element 
under torsional dynamic loading and investigated the microslip and macroslip phenomenon. Many other models were established to describe the stiffness of bolted joints, and the energy dissipations due to the friction between contact surfaces were also considered in a model whose parameters were determined by the corresponding experiments [9-11]. Such research concentrated on the behavior of the bolt under dynamic loading along the axial or tangential direction. Unfortunately, it is quite difficult to acquire the relative displacement of each bolt in the model needed for a flange joint, and this method may lead to low efficiency. Bolted joints appear different behavior at elevated strain rate [12]. Fransplass and Langseth took a thorough experimental and simulation study of bolted joints under tension or tension combined with shear stress at elevated strain rate, revealing the failure mode of joints $[13,14]$. Luan et al. [15] developed a bi-linear spring model validated for pipe structures with bolted flange joints. The structure introduced different stiffness under tensile and compressive stress, however, testing of the model under high frequency of excitation was not mentioned. Esteban and Rogers [16] established a model with spectral function conducting the energy dissipation of bolted joints. The research showed the response of joints and the energy dissipation were different under low and high frequency of excitation. Dynamic impacts can lead to a response covering a wide range of frequencies. In the signal processing of Wen and Zhou's [17] research, the experimental acceleration of penetration showed that most energy was distributed around the vibration mode of order 6-10. Nakalswamy [18] studied a joint under different preload with simulations and experiments. The fixtures were subjected to two levels of impacts using a hammer and a onestage gas gun, respectively. Grimsmo et al. [19] studied the behavior of bolted end-plate joints of steel under dynamic load with experiments and analyzed the relationship of failure mode and energy absorption. The research considering extreme impacts was the work of Somasundaram et al. [20]. A combined Lagrangian-SPH (Smooth Particle Hydrodynamics) approach was used to develop a finite element simulation that can capture both the physical damage of the structure and the shock propagation across the bolted joint. The author paid much attention to the reproduction and did not focus on the transmission and the characteristic vibration of the structure.

Numerical simulation is the main method used to analyze the behavior of bolted flange joints at present. Previous efforts offered a number of references. Kim et al. [21] developed four kinds of models, which are solid-bolt model, coupled-bolt model, spider-bolt model and no-bolt model. All the proposed models took into account pretension effect and contact behavior between flanges being joined. Those models were investigated under static loading and the accuracy was not determined under impact load. Schwingshackl et al. [22] studied the nonlinear behavior of bolted flange joints using a 3D numerical model validated with experimental hammer impacts. The nonlinear elements introduced a new thermal stress field calculated from the relationship of strain-preload. Although the contact forces between the bolts and flange were depicted thoroughly, an additional field will need more solving time. Semke et al. [23] confirmed that effective analysis techniques for 3D modeling were recommended to assess the influence of a bolted flange with an elastic gasket. The first two modes showed a very acceptable relationship between the experimental and simplified model results. It is highly possible to adopt this model in analyzing bolted flange joints under dynamic impact.

In this paper, a revised theory based on spectrum function is carried out to describe the dynamic response of bolted flange joints under impulse. Accordingly, experiments are practiced to validate the model, and the results show satisfactory agreement. Several half-sine impact waves were loaded in the model and the relationships of response and excitation are discussed. The regulation confirms that impact load can excite structural vibration and shock response; meanwhile, the discontinuous interfaces result in wave distortion and dissipation. This research offers an understanding of the local behavior of joints and is helpful for optimizing structural design under impact loading.

\section{Experimental setup}

A revised SHPB (Split Hopkinson Pressure Bar) experiment was used to validate the numerical models as shown in Fig. 1. The configuration consisted of four parts: the projectile, the incident bar, the assembled bolted flange joints and the absorber. The lengths of the striker bar were $0.1 \mathrm{~m}, 0.2 \mathrm{~m}$ and $0.3 \mathrm{~m}$ respectively, and that of the incident bar was $2 \mathrm{~m}$. All of them had a diameter of $37 \mathrm{~mm}$. The striker was driven by a compressed air gas-gun with a range of velocity from 3 to $7 \mathrm{~m} / \mathrm{s}$. Those loading conditions kept the joint under elasticity. The flange was connected by six M4 bolts to one end of the incident bar. Although the flange was fixed to the bar, its suspended body could still vibrate along the axial direction subjected to a dynamic impact loading. This procedure allowed for multiple, repeatable experiments to be conducted with a single testing apparatus.

A series of rectangular impact waves with different pulse widths were generated and propagated through the bolted flange joint. Strain gauges (BE120-1AA) were placed onto the incident bar and the suspended body of the flange as in Fig. 2. Signals were collected using a Wheatstone bridge circuit, a differential amplifier (Tektronix $5 \mathrm{~A} 22 \mathrm{~N}$ ), and a digital oscilloscope (Tektronix TDS 420) during the tests. All of the signals were measured as micro-strain and the sensitivity of the strain-gauge was 1000 micro-strains per volt. The strain signal was measured for $5 \mathrm{~ms}$ at a sampling rate of $2 \mathrm{MHz}$.

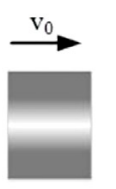

Striker

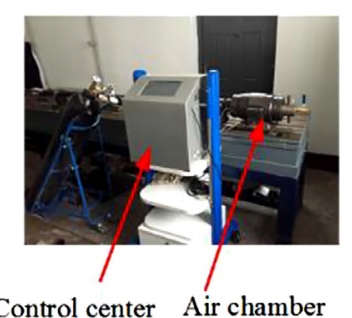

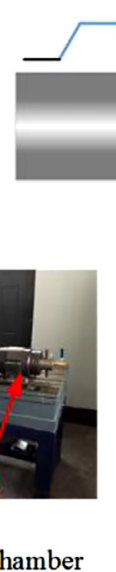

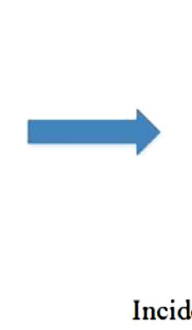

Incident bar

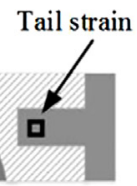

flange
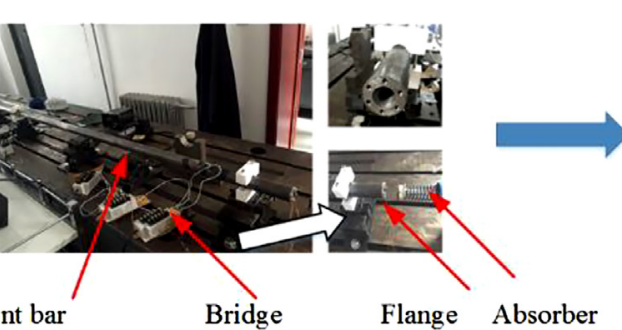
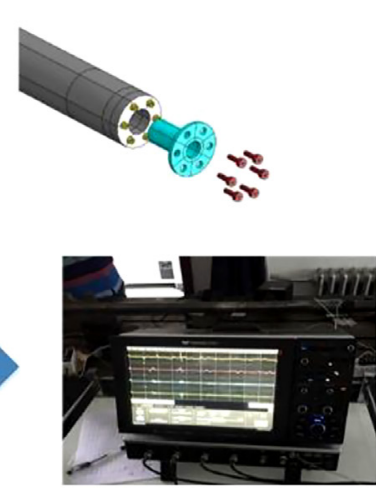

Oscilloscope

Fig. 1. The scheme of the revised SHPB (Split Hopkinson Pressure Bar) setup. 


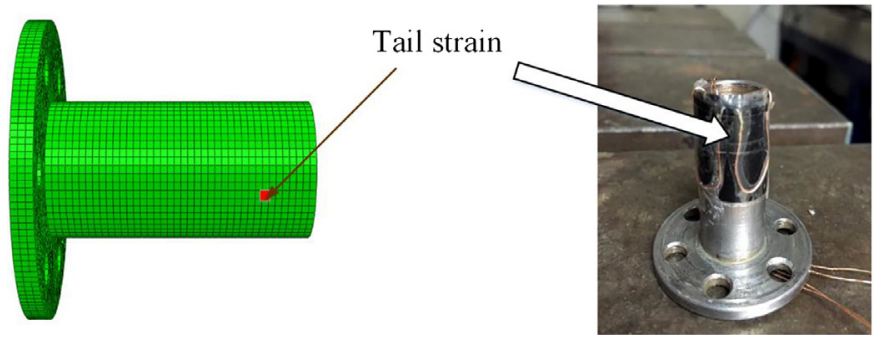

Fig. 2. The chosen element for comparison between the simulation and experiment.

\section{Theoretical analysis}

A bolted flange joint includes a flange mounted to one end of a bar by six bolts, as shown in Fig. 3. Stress waves will spread across several interfaces from the incident bar to the flange, as shown by the arrow. It is important to establish a method to model the interfaces and their corresponding dynamic response.

A revised theory is applied to describe vibrational characteristics of flanges under impact loading. According to research by Esteban et al. [24], the spectral formulation was introduced to predict the wave propagation and energy dissipation in joints. It transformed the solving procedure from the time to the frequency domain. This method, also as a dynamic stiffness matrix formulation, treated the distribution of mass and rotational inertia exactly, extending each element from discontinuity to discontinuity. For simplicity, only one dimension is considered in this analysis. The displacement assumes the following spectral representation:

$u(x, t)=\sum_{n} U_{o n} \exp \left[-i\left(k_{n} x-\omega_{n} t\right)\right]$

where $n$ is the particular mode, $U_{0}$ is the wave amplitude, $k$ is the wavenumber (1/length) and $\omega$ is the radial frequency of the wave (radians/time). For each mode $n$, the dispersion relation, which relates $k$ and $\omega$, is determined. Once the vibration modes of structure are confirmed, their amplitudes can be calculated for various sources of excitation.

According to the axial governing equation, the longitude displacement is satisfied with:

$E A \partial^{2} u / \partial x^{2}=\rho A \partial^{2} u / \partial t^{2}$

The solution in the frequency domain is presented as:

$u(x)=C_{1} e^{-i k x}+C_{2} e^{i k x}$

where the spectrum relation is given by $\mathrm{k}=\omega \sqrt{\rho / E}$. For each mode $n$, the coefficient can be determined exclusively. The axial force is defined as:

$F=E A \partial u / \partial x$
The solution and external force can be written in another way as:

$u=\mathbf{R}(x, \omega)\left(\begin{array}{l}C_{1} \\ C_{2}\end{array}\right)$

$F=\mathbf{R}(x, \omega)\left(\begin{array}{l}C_{1} \\ C_{2}\end{array}\right)$

The vector of coefficients is solved on the basis of the displacements and forces at the ends of each element. The local stiffness matrix is obtained from $\{F\}=[\boldsymbol{k}]\{u\}$. After obtaining the local stiffness matrix at each frequency, the global stiffness matrix $[\boldsymbol{K}]$ is assembled. The governing equation can be expressed as:

$[\boldsymbol{K}]\{u\}=\{F\}$

The nonlinear joint model was discussed in Esteban's research [24]. Since the friction and damping was the main source of nonlinearity, a dashpot modeled the viscous damping in the joints, while a spring provided the appropriate rigidity for the connection. The equation of motion describing the nonlinear vibration of a bolted structure due to a harmonic forcing function is given by:

$[\boldsymbol{K}]\{u\}+\left\{F_{N L}\right\}=\{F\}$

where $\left\{F_{N L}\right\}$ is the vector of local nonlinear internal forces concentrated at the joint. Similarly, the $\left\{F_{N L}\right\}$ is also expressed as a Fourier series under forced vibration:

$\left\{F_{N L}\right\}=a \cos \omega t+b \sin \omega t=K_{e q} \bar{u}+\eta_{e q} \dot{\bar{u}}$

The stiffness and damping of joints is equivalent to that of the interfaces. Therefore, the nonlinearity of the structure can be constituted with $K_{e q}$ and $\eta_{e q}$. The friction and damping is transferred into internal forces with the same radial frequency of Eq. (1). The detailed method for determining the parameters in Eq. (8) can be found in Esteban's research [24].

The response of axial strain can be simplified in Eq. (10). It consists of three terms, representing three parts of physical phenomenon, structural vibration, forced vibration and shock response. Those three terms can be acquired from Eq. (8). The radial frequency of structural vibration and shock response is the general solution of Eq. (8), while the other is the special solution:

$\frac{A}{A_{0}}=A_{d} \sin \left(\omega_{d} t / \alpha\right)+A_{n} \sin \omega_{n} t+A_{s} \sin \omega_{s} t$

$\omega_{d}=\pi / T_{d}, \omega_{n}=2 \pi / T_{n}, \omega_{s}=2 \pi / T_{s}$.

where $T_{d}$ is the duration of excitation, $T_{n}$ is the natural period and $T_{S}$ is the period of shockwave in the flange, and $\omega$ corresponds to the radial frequency. The dynamic response is evaluated by the duration and amplitude.

The force transmission ratio $\alpha$ in Eq. (10) appears in the forced vibration. In Luan's research [15], the FFT results of the flange deviated from the base frequency during forced vibration, indicating the existence of $\alpha$. However, it lacks further explanation of the
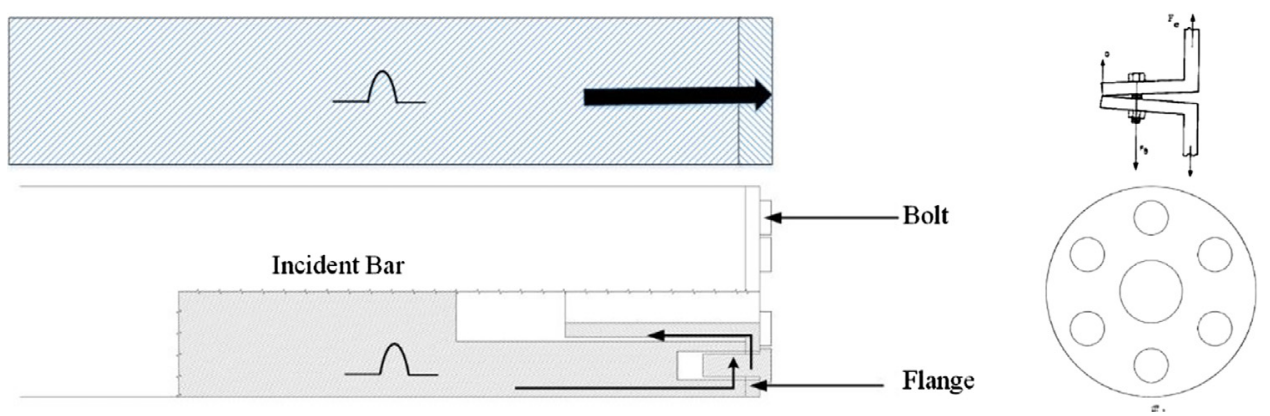

Fig. 3. The structure of bolted flange joints. 
mechanism at work [25]. Responses of linear systems can be regarded as a real-time response, that is, the frequency of response remains the same. The radial frequency of bolted flange joints is shorten under dynamic impulse because of complex interfaces.

\section{Numerical model}

The commercial FE (Finite Element) program ABAQUS 6.14 explicit was applied to model and analyze the assembled bolted flange joints subjected to dynamic impact loads. The FEA model had three components: the incident bar, the flange and the connecting bolts. The incident bar was a 2-m column bar with a diameter of $37 \mathrm{~mm}$. As shown in Fig. 4, there was a concentric hollow cylinder at one end of the bar along its axis. The hollow cylinder had a diameter of $17.6 \mathrm{~mm}$ and was $51 \mathrm{~mm}$ long. Four key geometric parameters were used to define the flange: the diameter of the flange, the height of the round plate, the length of suspended body and the thickness of the suspended cylindrical body, which were $37 \mathrm{~mm}, 2.56 \mathrm{~mm}$, $31.84 \mathrm{~mm}$ and $5 \mathrm{~mm}$, respectively. The round plate of the flange also consisted of six holes for bolts, and the holes were slightly larger than the bolts ( $5 \mathrm{~mm}$ ).

A 3D structural solid element was used for modeling the bolted flange joints, including the bar, the flange and the bolts. The element was defined by eight-nodes, having all six degrees of freedom at each node. Table 1 shows element size and number of elements for each component. It should be noted that elements of the connecting area must kept consistent so that the contact condition could be maintained.

To enhance the efficiency of the numerical model, six M4 bolts were created without screw threads along their rods. Instead, the part of screw area was substituted by a sleeve, namely, a connector, cut from the rod, as shown in Fig. 4. Thus, they consisted of round caps representing the bolt head, the rods and the sleeve. In addition, the diameter of the caps was $6 \mathrm{~mm}$ and the thickness of the sleeve was $0.4 \mathrm{~mm}$, the same as the clearance of the M4. The damping of the sleeve is defined by Rayleigh's theory. The mass damping has little effect on the low-order vibration and thus can be ignored [26]. The coefficient of material damping is defined according to the test result.

Contacts between the two pairs (bolts and connector, connector and bar) adopted tied constraints. General contact was defined at all the surfaces which might contact excluding the tied surface above, though the flange surface remained free. Different types of input loads were applied to the other end of the bar (without the flange). In all numerical cases, a modulus of elasticity of $210 \mathrm{GPa}$ and a Poisson's ratio of 0.3 were used for the incident bar. The flange was also made of steel with a modulus of $200 \mathrm{GPa}$ and the same Poisson's
Table 1

Lagrangian element size and numbers.

\begin{tabular}{lll}
\hline Part & Element size $(\mathrm{mm})$ & No. of elements \\
\hline Incident bar & 5 & $2,157,178$ \\
Flange & 0.8 & 11,679 \\
Connector & 0.3 & 13,500 \\
Bolt & 0.4 & 24,732 \\
\hline
\end{tabular}

Table 2

Comparison of the stress wave in the incident bar.

\begin{tabular}{lllll}
\hline & \multicolumn{2}{l}{ The incident stress wave } & \multicolumn{2}{l}{ The reflected stress wave } \\
\hline & Experiment & Simulation & Experiment & Simulation \\
Pulse width $(\mu \mathrm{s})$ & 144 & 139.5 & 159 & 150 \\
Maximum stress $(\mathrm{MPa})$ & 190.62 & 199.36 & 187.05 & 189.84 \\
Time for ascending $(\mu \mathrm{s})$ & 32 & 31 & 56 & 57 \\
\hline
\end{tabular}

ratio as the bar. The strength of connectors replacing the contact area were $2 \mathrm{GPa}$ and had a beta damping factor $\left(5 \times 10^{-5}\right)$ restraining high frequency in the simulation.

\section{Results and discussion}

\subsection{Validity of the numerical model}

In this section, the simulation model is validated. The stress waves in the incident bar are evaluated, including the incident and reflected waves. To remove high-frequency noises, the stress and axial strain were low-pass filtered at $80 \mathrm{kHz}$, which is slightly higher than the frequency of shock response in the flange. The material damping is tuned to 0.00005 to match the experimental result.

Table 2 indicates the favorable agreements between the experiments and the simulation. The energy of the incident wave and the reflected wave of the experimental case and simulated model are $6.83 \mathrm{~J}, 6.52 \mathrm{~J}, 6.81 \mathrm{~J}$ and $6.55 \mathrm{~J}$, respectively, as seen in Fig. 5. Fig. 6 shows the FFT spectrum of both the experimental and numerical results. Good agreement at the base frequency point confirm that the flange is at the same physical state. As expected, it can be seen that the simulation model is validated for analyzing bolted-flange joint structures under impact loading.

\subsection{The strain of a flange and its FFT spectrum}

Half-sine waves with a wide range of time widths were applied to the model. The amplitude of excitation was $120 \mathrm{MPa}$, ensuring the response was under an elastic state. Those excitations covered a

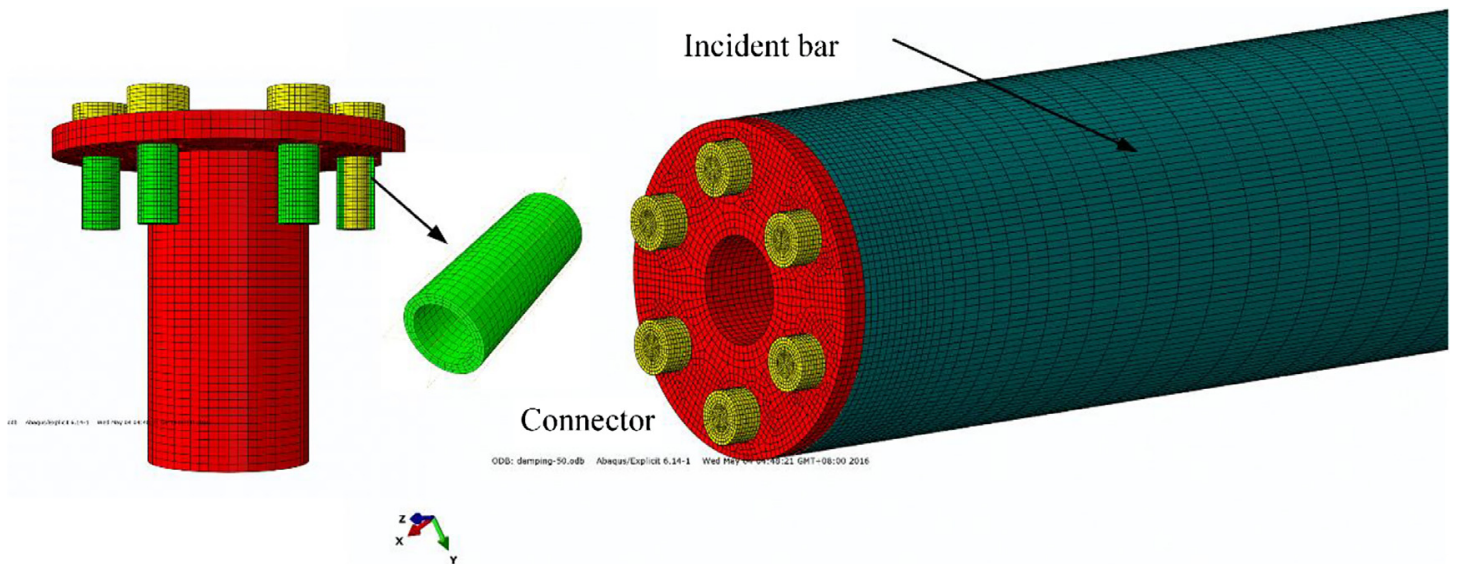

Fig. 4. The simulated model of a bolted flange structure. 


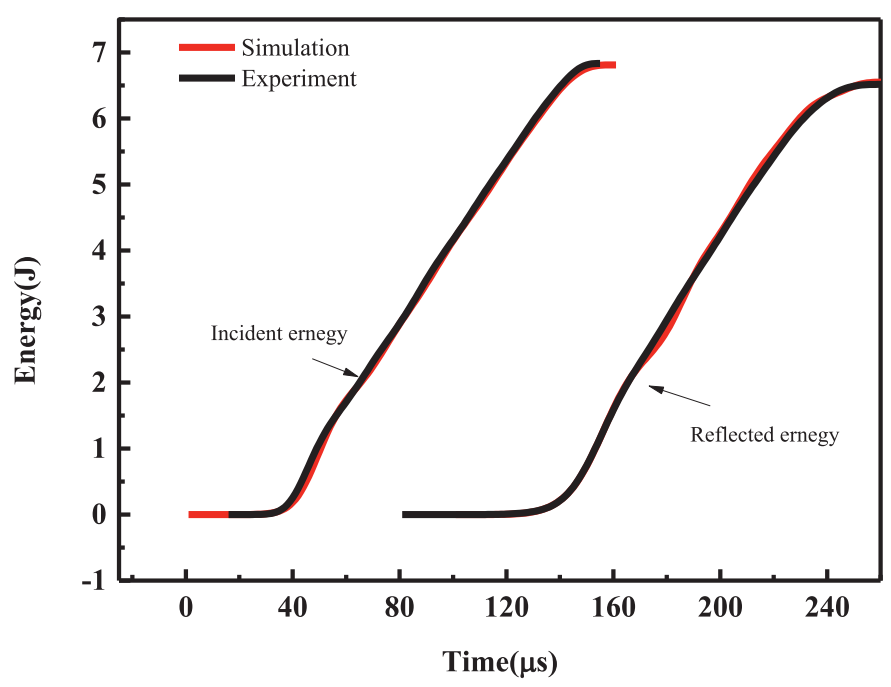

Fig. 5. Comparison of the incident and reflected energy in the incident bar.

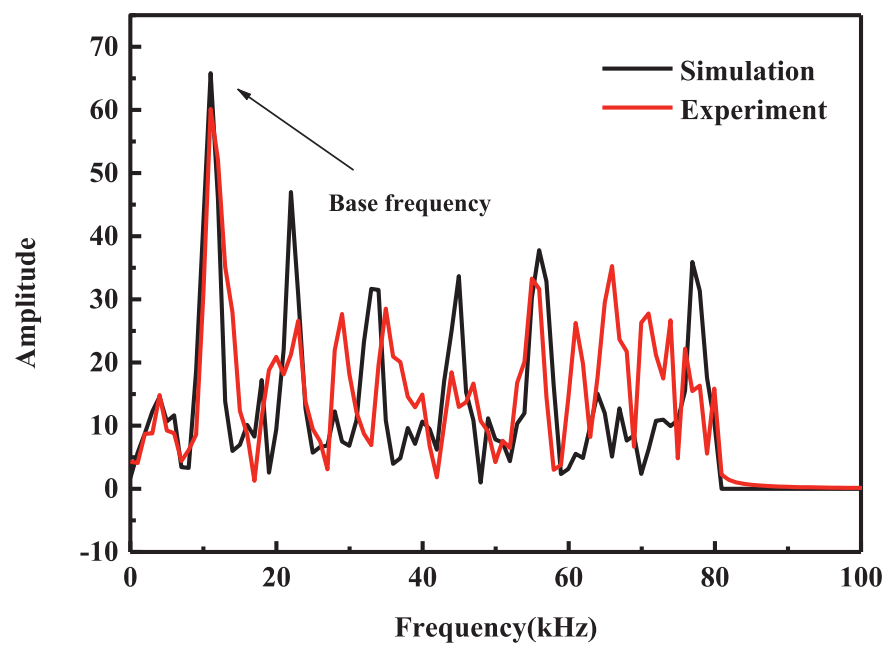

Fig. 6. Comparison of the FFT spectrum of axial strain in a flange under square impulses. range of $20 \mu \mathrm{s}-200 \mu \mathrm{s}$ with an increment of $10 \mu \mathrm{s}$. The energy dissipation exhibited different mechanisms for high frequency excitation. The highest frequency was set at over twice the natural frequency of the bolted-flange structure and the response of strain under different impact loading was evaluated.

The analysis focuses on the response of the flange during the entire progress of impact loading and unloading. Fig. 7 shows a typical response of a bolted flange joint under impact loading. Similar to the linear system, the signal contains two regions, namely, the impact response region and the vibrational response region, which represent forced vibration and damping vibration, respectively. Passing through the interfaces of the joint, a shockwave will dissipate and reflect, and as a result, the pulse width will increase. This change in response will be detected in reflected waves of the incident bar. Therefore, the pulse width of the reflected wave is defined as the duration of the impact response region. The beginning and end of the wave also require definition. The shockwave has to spread $373 \mu$ s to reach the interface of the flange, with that time used as the starting point. According to the classic theory of vibration, the change in amplitude of a signal is fitted to an exponential function. Therefore, the end point is decided by the damping curve of the vibrational response region. The envelope of the noises is presented in Fig 7, and the intersection of the damping curve and envelope is defined as the end point. For instance, the end point in Fig 7 is the fourth highest peak of the vibrational response region.

The FFT spectrum of the response is also evaluated. Fig. 8 shows the FFT spectrum of axial strain in a flange under a $35 \mu$ s semi-sine impulse, where most energy concentrates on the base frequency of $11 \mathrm{kHz}$. There are some peaks at the integral multiple of base frequency; all of the peaks together with the peak at base frequency represent the structural vibration. Additionally, a higher frequency of approximately $74 \mathrm{kHz}$ is detected. The corresponding period of that frequency is the spreading duration of the shockwave in the flange. This indicates that intense excitation can excite not only structural vibration but also a shock response.

The responses of different regions are discussed. The Fig. 9 gives the curve of reaction ratio in the impact response region. The reaction ratio is the scale of axial strain to loading. Only two components of the frequencies were observed in the signal. As Fig. 9 shows, the low-frequency signal is the low pass filter of $11 \mathrm{kHz}$, describing the effect of the excitation on the joint. The other frequency is the high

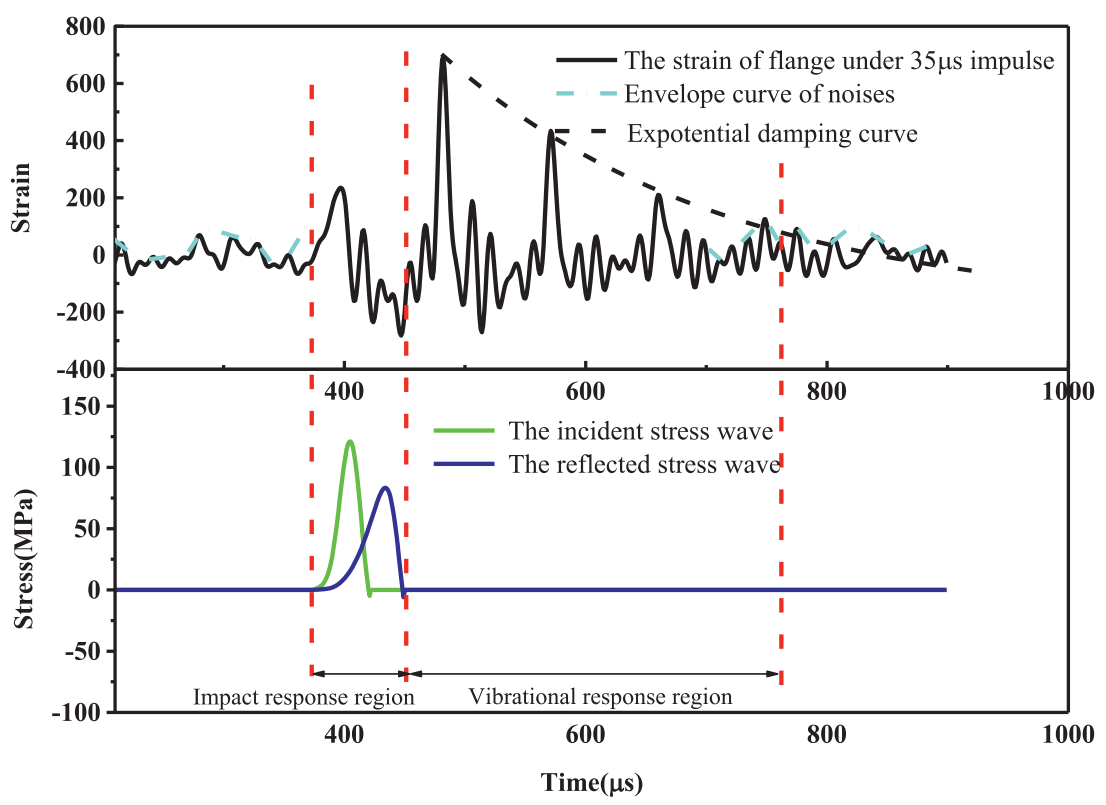

Fig. 7. The typical axial strain of a flange under a $35 \mu$ s half-sine impulse. 


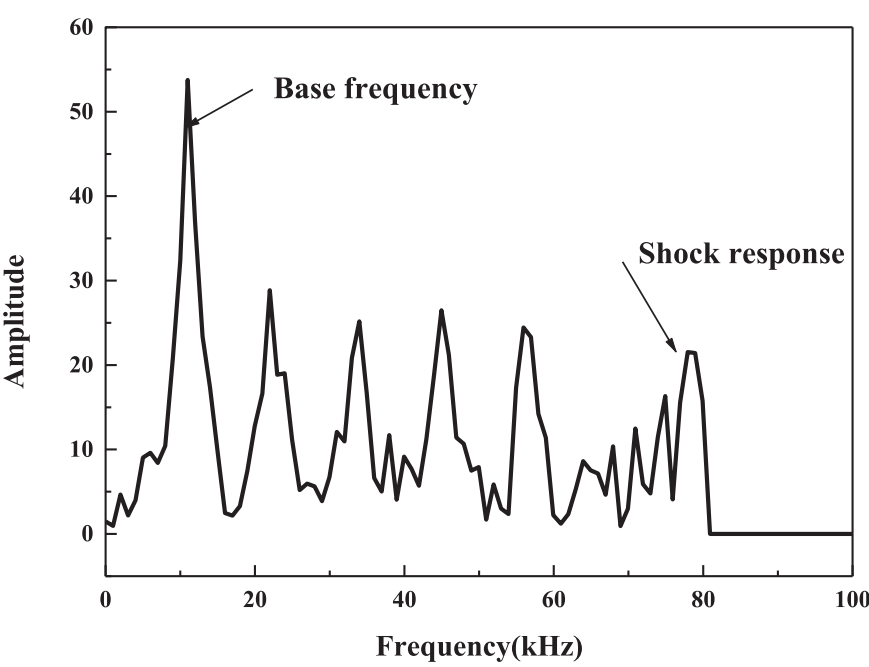

Fig. 8. The FFT spectrum of axial strain in a flange under a $35-\mu$ s semi-sine impulse.

pass filter of $70 \mathrm{kHz}$, where the period of impact waves in the flange is approximately $13 \mu \mathrm{s}$. This signal exists throughout the impact response region and the peaks are nearly the same. The linear system under dynamic load only vibrates at the natural and input frequency. However, the nonlinear system excites a shock response. In Fig. 10, the reaction ratio and component of the frequency in the vibrational response region is shown, where the signal of the vibrational response region contains two parts of the frequency. The low frequency is approximately $11 \mathrm{kHz}$, which is the natural frequency. This conforms to the vibrational theory of a linear system under impact loading. The signal remains damping with a period of $90 \mu \mathrm{s}$ until the energy is expended. Apart from that, the high frequency was also the high pass filter of $70 \mathrm{kHz}$, which means that shock response exists throughout the region. It can be concluded that only under extreme impact loading can high order vibration be excited in bolted flange joints.

\subsection{The force transmission ratio}

This section investigates the change in frequency. The impulse widens when passing through the contact interfaces. Shockwaves dissipate and reflect once arriving to the free surfaces. Furthermore, the restriction condition of the joint is neither fully cohesive nor free. Instead, the areas of contact surfaces are always changing. The complex geometry of bolts and their stiffness with damping increase the nonlinearity. The frequency of the signal is lower than the original one, that is, all the reflected waves have larger pulse widths than the incident ones. Therefore, the force transmission ratio $\alpha$ describes the change in radial frequency.

Acquiring further laws from the trend in $\alpha$, different durations of the impact response region under various time widths of half-sine waves were conducted. As shown in Fig. 11, all the parameters need to be dimensionless. The horizontal axis is dimensionless loading time, the pulse width of excitation $T_{d}$ to the natural period $T_{0}$, while the vertical axis represents response, the duration of impact response region $T_{p}$ to the pulse width $T_{d}$.

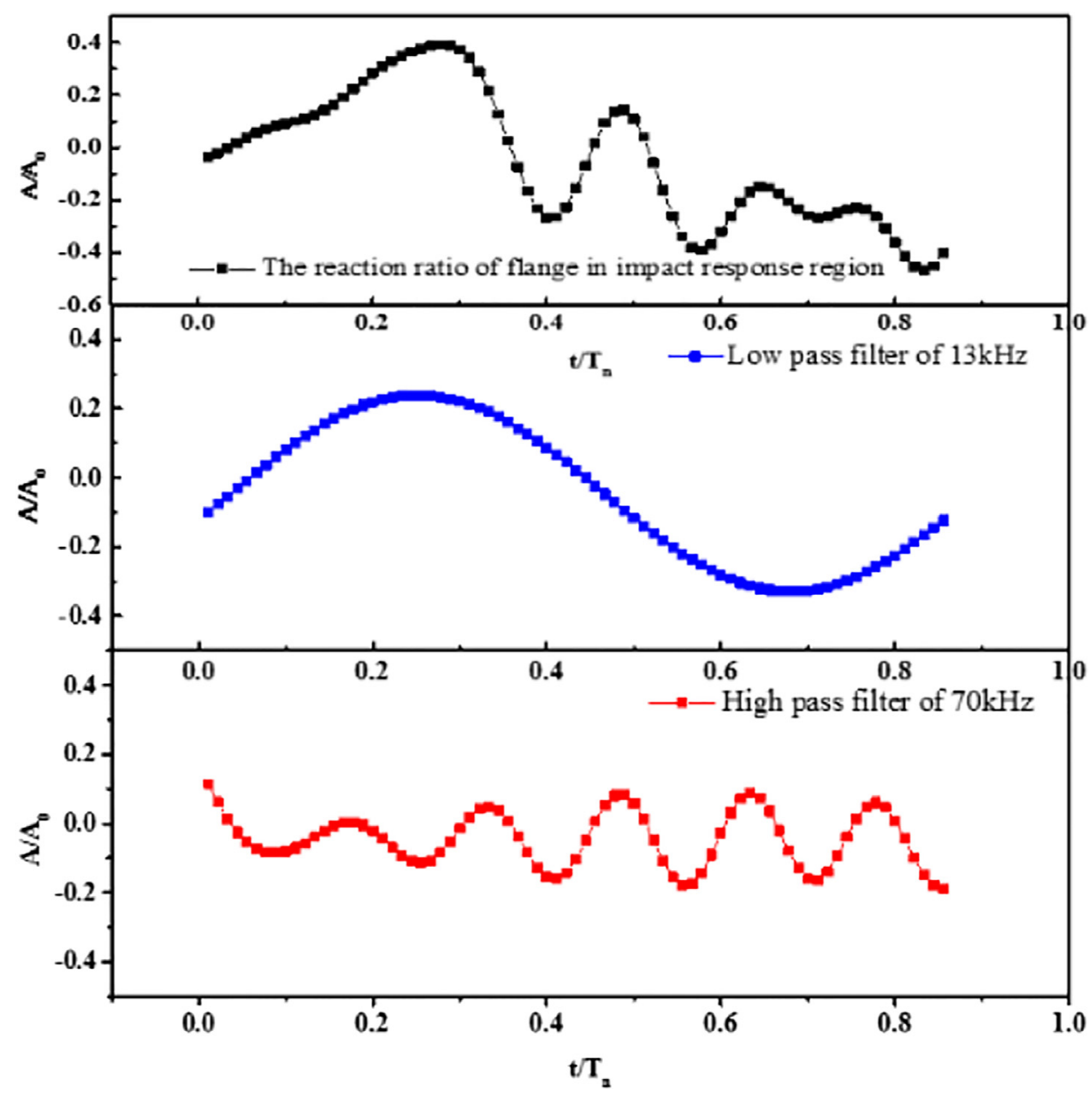

Fig. 9. The reaction ratio and components of the frequency of the impact response region. 


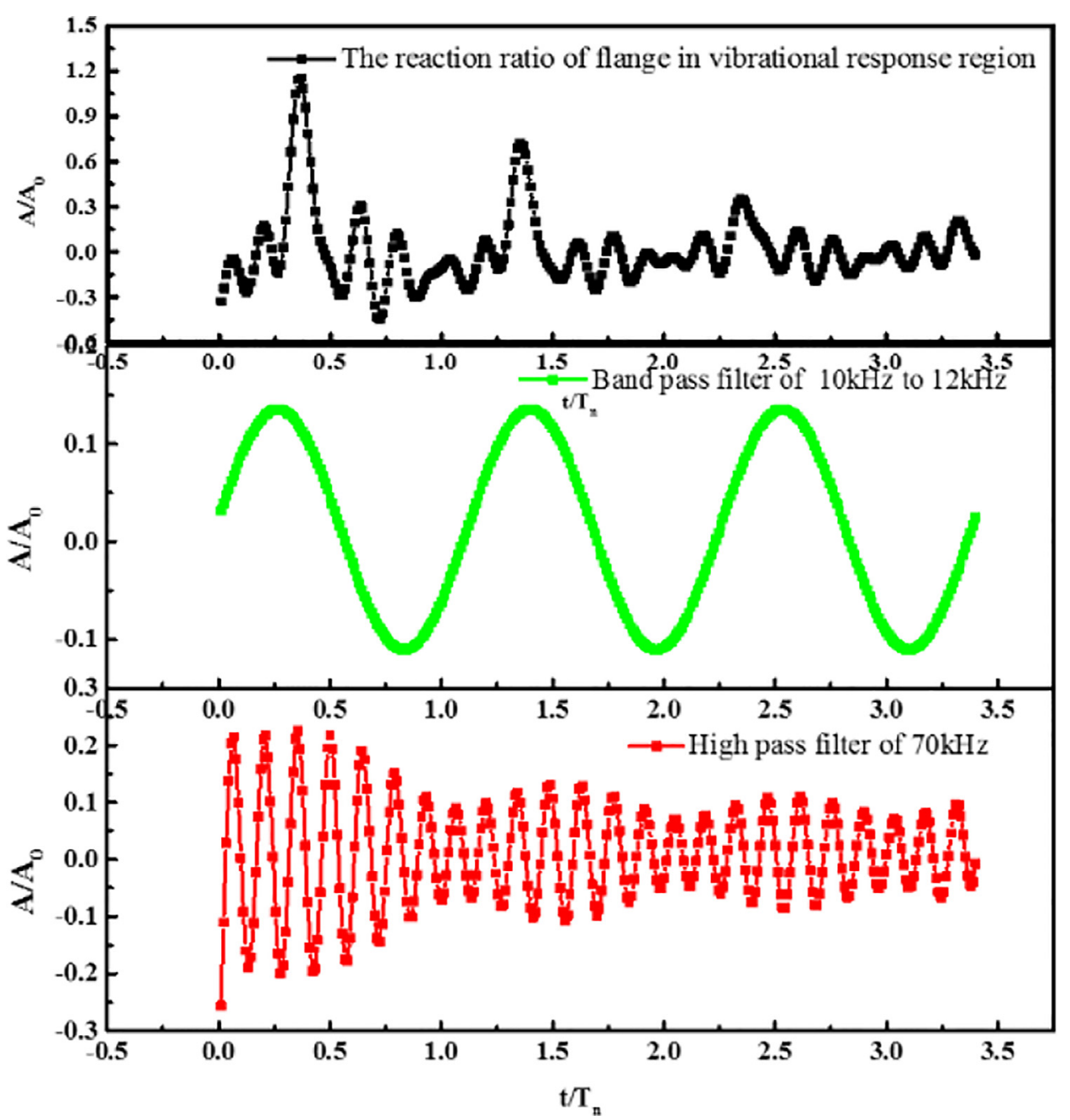

Fig. 10. The reaction ratio and component of the frequency in the vibrational response region.

The relationship between the response and excitation is shown in Fig. 11. The higher input frequency led to a longer duration of impact response region. The pulse widths for the incident and reflected waves are almost the same for low-input

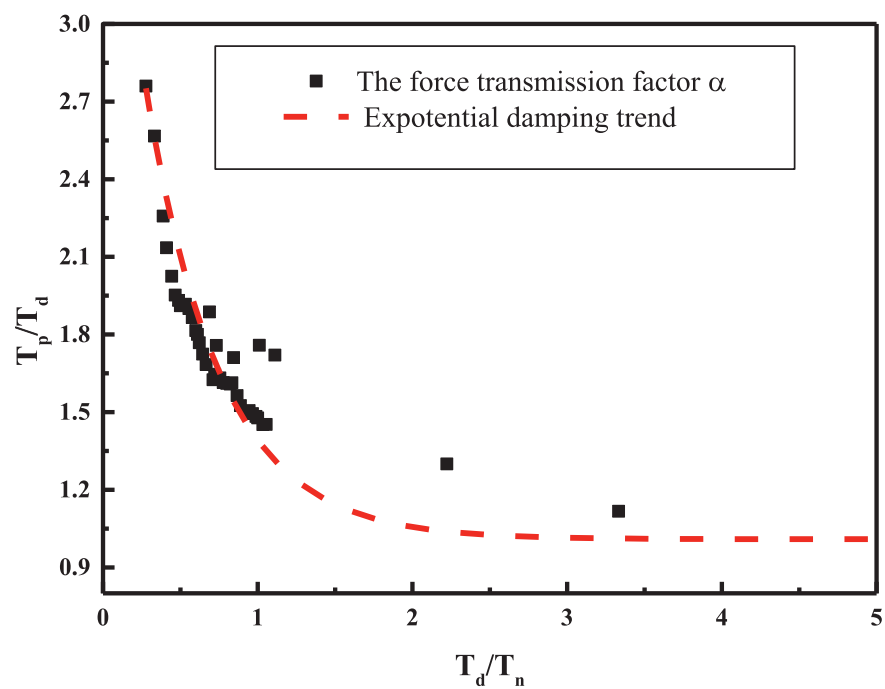

Fig. 11. The duration of impact response region to loading under different dimensionless pulse widths. frequencies of approximately $90 \mu \mathrm{s}$, which conforms to the theory of linear systems under half-sine impulse loading. It should be noted that the shock response is prominent under intense excitation. The effect of the loading condition on $\alpha$ is reduced as the frequency decreases. Ultimately, as Fig. 11 shows, a longer impulse is regarded as a quasi-stationed load on the joint and the duration of the impact response region nearly equals the excitation. In conclusion, the effectiveness of the force transmission ratio is limited by the loading condition. It is more important under short-pulse impacts, and when the joint is under mild excitation, the response follows the rule of linear systems under dynamic impacts. Although the effect of intense excitation is reduced, nonlinearity from surfaces in joints still exists, and the ratio of $T_{p}$ to $T_{d}$ continues to be larger than 1 . Therefore, the revised model for the duration of the impact response region has a limited condition and is better suited for short impulses.

\subsection{Damping rate of energy}

Amplitude is another parameter used in evaluating the vibration in both regions. In Fig. 12, A denotes the amplitude of the axial strain, while $A_{0}$ denotes the loading strain. As for theoretical analysis, the amplitude is decided by the proportion of the coefficient in different terms. When the loading time is half of the natural period, the joint will resonate and the amplitude is at its highest. The energy of vibration becomes smaller as the frequency of excitation gradually reduces. The short impulse will generate high-amplitude 


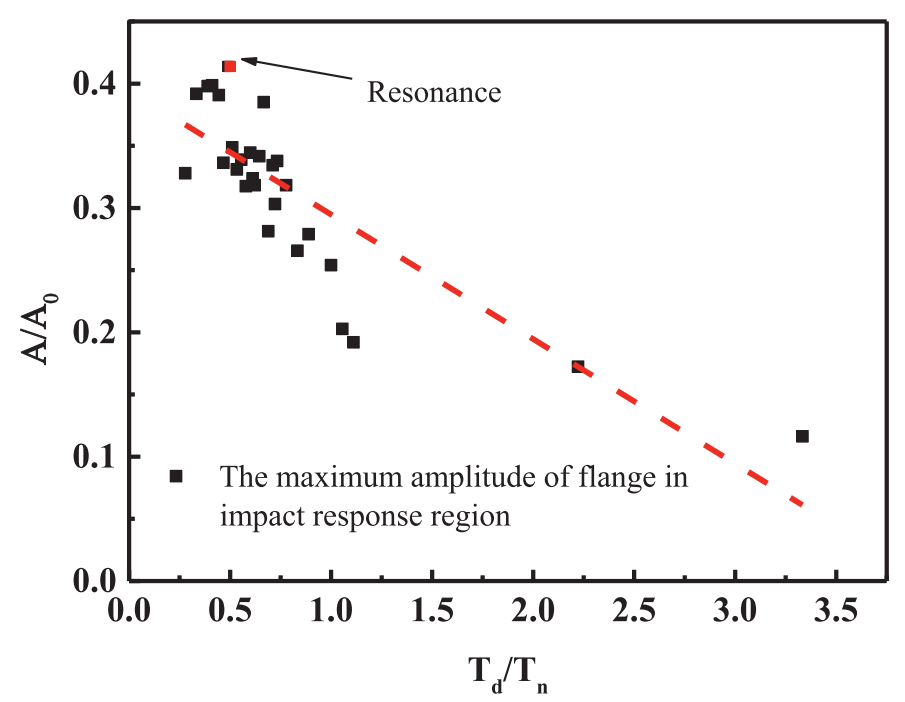

Fig. 12. The maximum amplitude of a flange in the impact response region.

vibrations in the impact response region. The amplitude of the vibrational response region is defined by the maximum peak of response as seen in Fig. 13. The energy received is reduced (Fig. 14) when the frequency of the excitation decreases. Consequently, the amplitude is limited by the decreasing function. The vibration cannot be fully understood with only one peak, and as a result, the damping rate is also determined to explain the response under different impact loading.

The damping curve is the change in peaks in the vibrational response region. In Fig. 15, a higher frequency of excitation leads to a smaller rate of damping. The stress of the bar in Fig. 16, after the reflected wave, implies that there remains a small amount of energy passing through the joint from the flange to the bar. Fig. 14 shows the percentage of flange energy relative to the loading. The energy of the flange, which is the remainder between incident and reflected energy in the bar, is continuously receding. When the pulse width of excitation is over $90 \mu \mathrm{s}$, the percentage keeps decreasing to under $5 \%$. Part of the energy received of the flange under intense excitation dissipates because of structural damping, and the remainder is transferred out of joint. One explanation for this is that the joint under impact loading is more likely to form a prying force and the

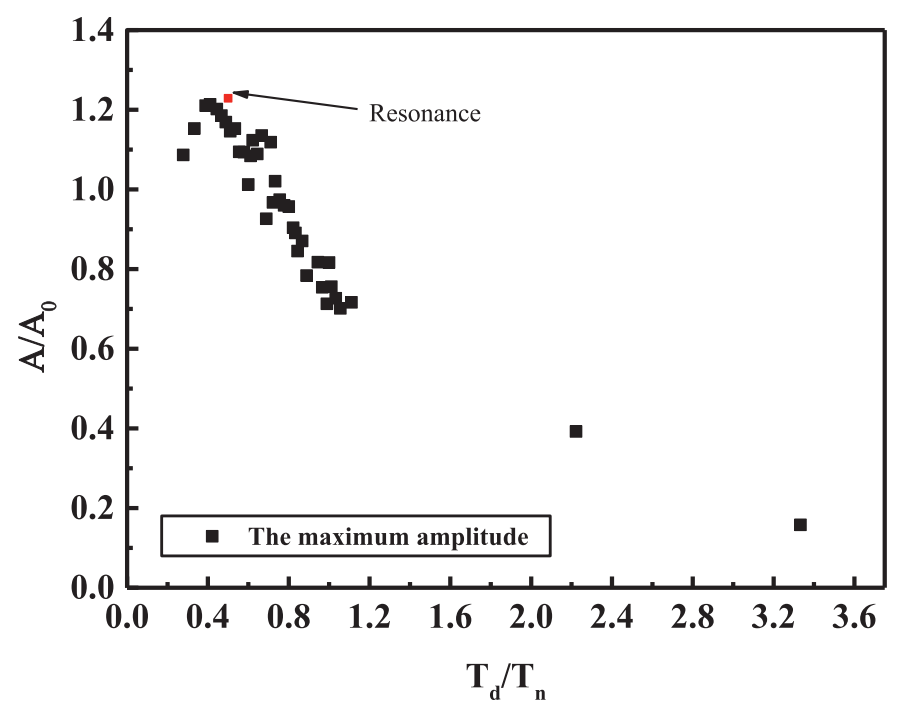

Fig. 13. The maximum amplitude of a flange in the vibrational response region under different dimensionless pulse widths.

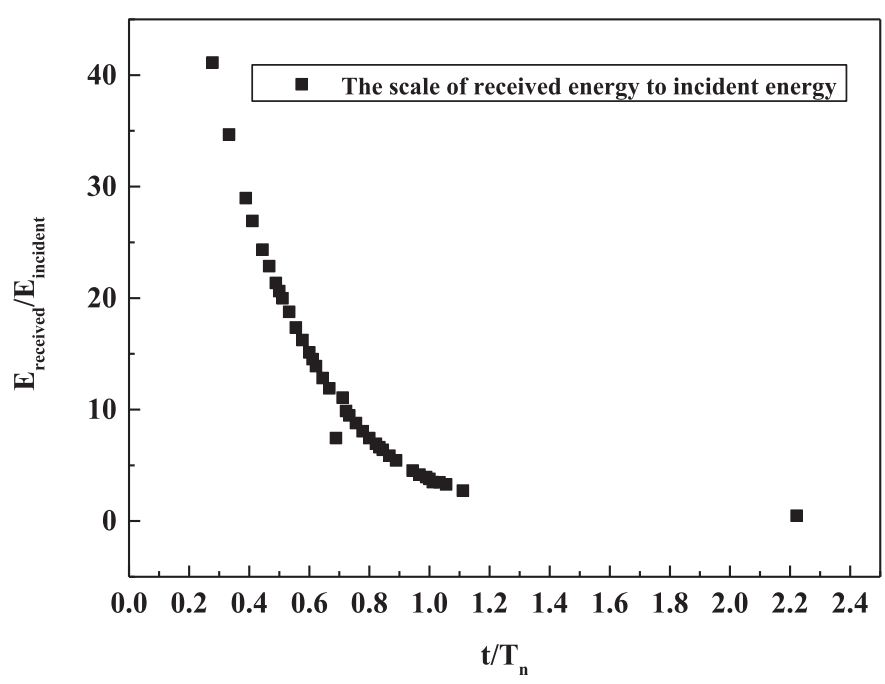

Fig. 14. The energy received of a flange under different dimensionless pulse widths.

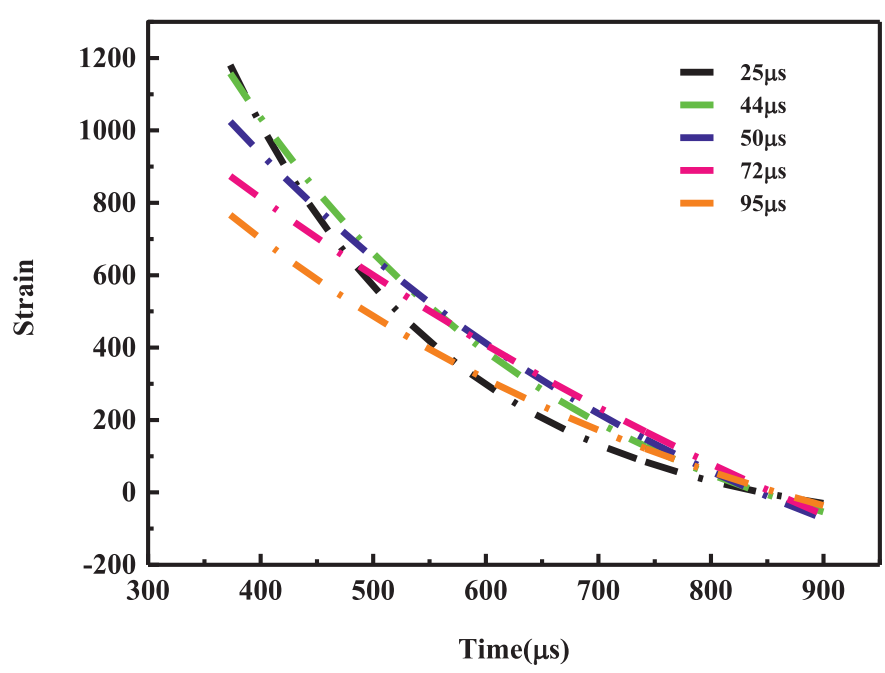

Fig. 15. The damping rate of the vibrational response under several typical impulses.

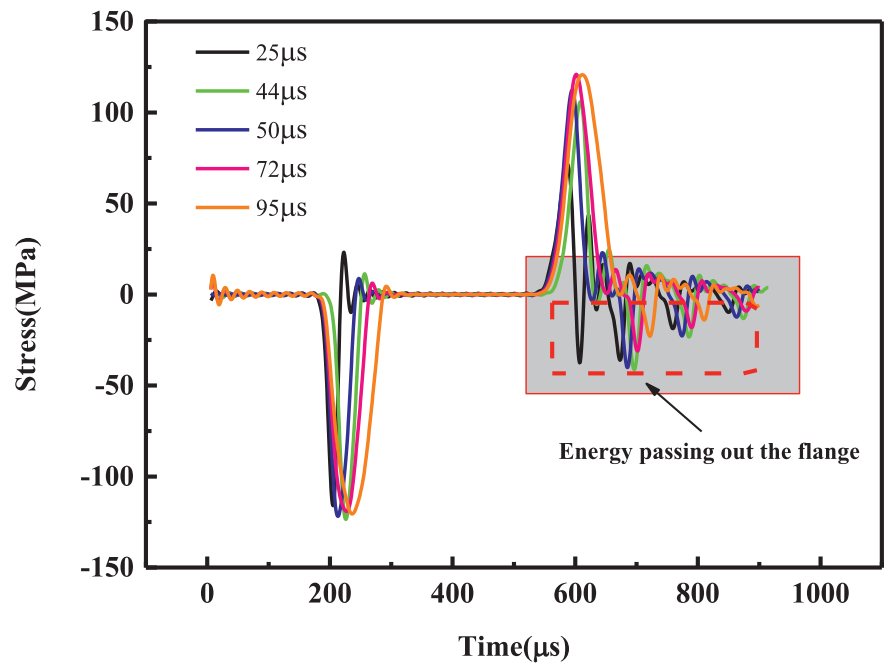

Fig. 16. The stress wave in the incident bar under several typical impulses. 


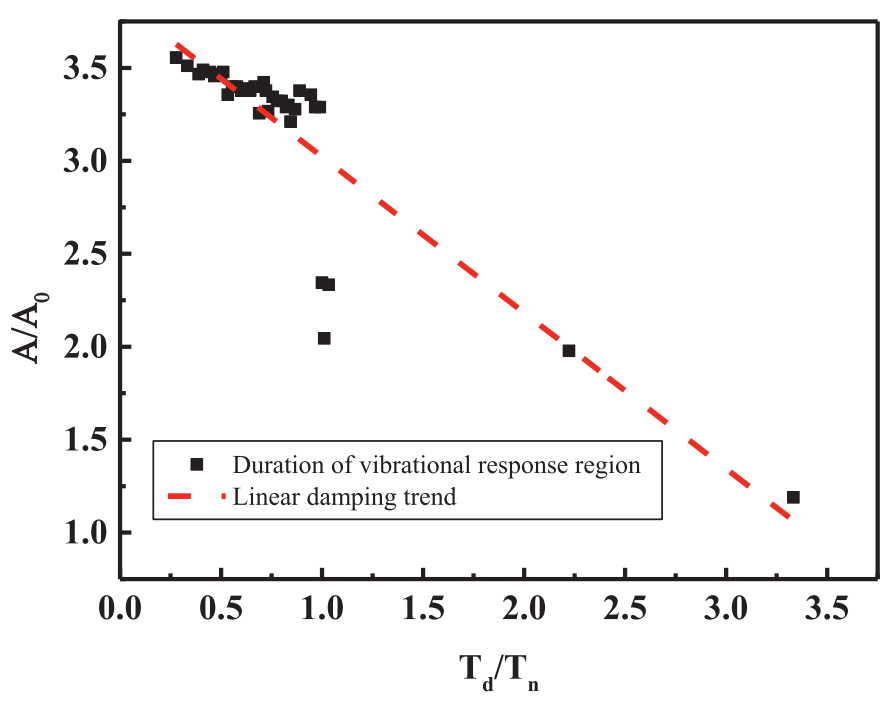

Fig. 17. The duration of the vibrational response region under different dimensionless pulse widths.

contact interfaces are not uniformly connected. Additionally, shockwaves continue spreading in the flange and can interact with the bar due to different local velocities.

The duration of the vibrational response region is also decreasing as the frequency of excitation decreases, as seen in Fig. 17. Since the damping vibration depends only on the energy received, the change of energy restricts the duration. Initially, the energy received at the flange is nearly half of the incident amount. When the joints experience longer impulses, such as $80 \mu$ s, the energy remaining at the flange is only $5 \%$. Although the amplitude is already lower as the frequency decreases, the duration also decreases.

\section{Conclusions}

The objective of this research was to study the force transmission and vibration characteristics of bolted flange joints under impact loading. A revised theory was proposed for describing the response of nonlinear systems under dynamic impact and for determining the components of frequencies, which is an important component of the responses. A 3D model was established to investigate the relationship between the excitation and response of the joints. The modified SHPB setup was used to validate the results of the simulation. The experimental and numerical results showed excellent correlation in natural frequency with square waves. Different pulse widths of half-sine waves in elasticity were applied to the model obtaining the law of force transmission. Bolts with damping and automatic contact interfaces with friction were substituted for the nonlinearity of joints. The results of the simulation were discussed, including the duration, amplitude and energy of response under various impact loads.

1. The simulated model was able to reproduce the response of bolted flange joints. The response was divided into two regions, the impact response region and the vibrational response region according to the pulse width of reflected waves in the incident bar. The model confirmed that the impact loading can excite structural vibrations and shock responses in both regions.

2. Discontinuous interfaces contributed to structural damping and nonlinear contact, leading to the distortion and dissipation of shockwaves. The frequency of response was changed from that of the excitation, which is characterized by the force transmission ratio $\alpha$. It was reduced from 2.8 and ultimately approached 1 due to the load condition, indicating that nonlinearity of joints would decrease as the frequency of excitation decreased.

3. Since the energy received on the flange continued to decrease, the amplitude in both regions also decreased. In addition, the amplitude reached its maximum value at the base frequency, approximately $11 \mathrm{kHz}$, where the structure resonated. Additionally, shock response was prominent under short-pulse waves. The damping rate of response was also investigated and higher frequencies of excitation were found to induce a large damping rate. There remained some energy transferring from the flange to the incident bar.

\section{Acknowledgments}

The authors would like to thank the National Natural Science Foundation of China [Grant no.11502274, 11002150, 11332011, 11402277] and the Basic Research Equipment Project of the Chinese Academy of Sciences [YZ200930] for financial support.

\section{Reference}

[1] Cao JJ, Bell AJ. Determination of lbolt forces in a circular flange joint under tension force. Int J Press Vessels Pip 1996;68:63-71.

[2] Bickford JH. Introduction to the design and behavior of bolted joints. 4th Ed. 2007.

[3] Blachowski B, Gutkowski W. Effect of damaged circular flange-bolted connections on behaviour of tall towers, modelled by multilevel substructuring. Eng Struct 2016;111:93-103.

[4] El-Zahry RM. Optimum design of preloaded bolted joint under harmonic excitation. J Sound Vibr 1986;108:455-70.

[5] Mackerle J. Finite element analysis of fastening and joining: A bibliography (1990-2002). Int J Press Vessels Pip 2003;80:253-71.

[6] Ibrahim RA, Pettit CL. Uncertainties and dynamic problems of bolted joints and other fasteners. J Sound Vibr 2005;279:857-936.

[7] Oldfield M, Ouyang H, Mottershead JE. Simplified models of bolted joints under harmonic loading. Comput Struct 2005;84:25-33.

[8] Ouyang H, Oldfield MJ, Mottershead JE. Experimental and theoretical studies of a bolted joint excited by a torsional dynamic load. Int J Mech Sci 2006;48:144755 .

[9] Vadean A, Leray D, Guillot J. Bolted joints for very large bearings-numerical model development. Finite Elem Anal Des 2006;42:298-313.

[10] Yu H, Burgess IW, Davison JB, Plank RJ. Numerical simulation of bolted steel connections in fire using explicit dynamic analysis. J Constr Steel Res 2008;64:51525.

[11] Schwingshackl CW, Petrov EP. Modeling of flange joints for the nonlinear dynamic analysis of gas turbine engine casings. J Eng Gas Turbines Power 2012;134:122504

[12] Fransplass H, Langseth M, Hopperstad OS. Tensile behaviour of threaded steel fasteners at elevated rates of strain. Int J Mech Sci 2011;53:946-57.

[13] Fransplass H, Langseth M, Hopperstad OS. Numerical study of the tensile behaviour of threaded steel fasteners at elevated rates of strain. Int J Impact Eng 2013;54:19-30.

[14] Fransplass H, Langseth M, Hopperstad OS. Experimental and numerical study of threaded steel fasteners under combined tension and shear at elevated loading rates. Int J Impact Eng 2015;76:118-25.

[15] Luan Y, Guan Z-Q Cheng G-D, Liu S. A simplified nonlinear dynamic model for the analysis of pipe structures with bolted flange joints. J Sound Vibr 2012;331:325-44.

[16] Esteban J, Rogers CA. Energy dissipation through joints: theory and experiments. Comput Struct 2000;75:347-59.

[17] Wen F, Zhou Z. Simulation and experiment of projectile penetrate into steel target acceleration signal processing. Int J Image Graph Signal Process 2011;3.

[18] Karpanan Nakalswamy K. Experimental and numerical analysis of structures with bolted joints subjected to impact load. Gradworks; 2010.

[19] Grimsmo EL, Clausen AH, Langseth M, Aalberg A. An experimental study of static and dynamic behaviour of bolted end-plate joints of steel. Int J Impact Eng 2015;85:132-45.

[20] Somasundaram DS, Trabia MB, O'Toole BJ. A methodology for predicting high impact shock propagation within bolted-joint structures. Int J Impact Eng 2014;73:30-42.

[21] Kim J, Yoon J-C, Kang B-S. Finite element analysis and modeling of structure with bolted joints. Appl Math Modell 2007;31:895-911.

[22] Schwingshackl CW, Di Maio D, Sever I, Green JS. Modeling and validation of the nonlinear dynamic behavior of bolted flange joints. J Eng Gas Turbines Power 2013;135:122504. 
[23] Semke WH, Bibel GD, Jerath S, Gurav SB, Webster AL. Efficient dynamic structural response modelling of bolted flange piping systems. Int J Press Vessels Pip 2006:83:767-76.

[24] Esteban J, Lalande F, Rogers C, Chaudhry Z. Theoretical modeling of wave propagation and energy dissipation in joints. Adapt Struct Forum 1996.
[25] Chopra AK. Dynamics of structures : theory and applications to earthquake engineering. Pretenci-Hall; 1995.

[26] A.I. Yusuf, N.M. Amin, Determination of rayleigh damping coefficient for natural damping rubber plate using finite element modal analysis, 2015. 